\title{
Distinct Patterns of Expression of Two VAMP Genes Within the Rat Brain
}

\author{
William S. Trimble, ${ }^{1}$ Thackery S. Gray, ${ }^{2}$ Lisa A. Elferink, ${ }^{1}$ Michael C. Wilson, ${ }^{3}$ and Richard H. Scheller ${ }^{1}$ \\ 'Department of Biological Sciences, Stanford University, Stanford, California 94305, '2Department of Anatomy, Loyola \\ University Medical Center, Maywood, Illinois 60153 and, ${ }^{3}$ Department of Neuropharmacology, Research Institute of \\ Scripp's Clinic, La Jolla, California 92037
}

\begin{abstract}
VAMPs are synaptic vesicle-specific proteins composed of a carboxy-terminal hydrophobic membrane anchor and an approximately $\mathbf{1 0 0}$ amino acid domain oriented towards the cytoplasm. In rat, two $77 \%$ homologous VAMP genes are expressed in the CNS. To precisely localize the neurons expressing these 2 forms of VAMP, we have used RNA blotting and in situ hybridization histochemistry with RNA probes specific for the $3^{\prime}$ untranslated regions of the transcripts. These experiments revealed that the 2 genes are expressed in distict, but slightly overlapping, patterns in the rat brain. VAMP-1 expression is localized to a limited number of nuclei, particularly those involved in modulating somatomotor functions, while VAMP-2 expression is more ubiquitous, being found in nuclei associated with autonomic, sensory, and integrative roles. These data suggest that the specific structural features of individual VAMPs may play an important role in synaptic vesicle metabolism.
\end{abstract}

Synaptic vesicles in all neurons undergo common processes such as axonal transport, nerve terminal docking, and $\mathrm{Ca}^{2+}$-stimulated exocytosis and membrane recycling (Kelly, 1988). It is not surprising, therefore, that a number of proteins have been identified which appear to be common to all types of synaptic vesicles (Matthew et al., 1981; Buckley and Kelly, 1985; Jahn et al., 1985; Wiedenmann and Franke, 1985; Obata et al., 1987; Lowe et al., 1988; Floor and Feist, 1989). On the other hand, cell-type specific functions, such as those required for release and reuptake of various classes of neurotransmitters. should require specialized components unique to particular vesicle subtypes.

Since relatively little is known about the molecular mechanisms which underlie chemical neurotransmission, it is important to characterize both general and specific components of synaptic vesicles. Previously we described the cloning and characterization of a cDNA encoding a vesicle-specific protein from Torpedo californica (Trimble et al., 1988). This protein is expressed specifically in the nervous system and is remarkable both for its 3-domain structure and for its small size. In partic-

\footnotetext{
Received Aug. 21, 1989; revised Oct. 18, 1989; accepted Oct. 24, 1989.

We wish to thank Dr. Robert Sapolsky for providing dissected rat brain tissue and Dr. Sue McConnell for critically reading the manuscript. W.S.T. is the recipient of a Centennial Fellowship from the Medical Research Council of Canada. This work was supported in part by NIH grant NS 23038 to M.C.W., grants from the NIMH and NSF to R.H.S., and NIH grant NS 20041 to T.S.G.

Correspondence should be addressed to Dr. Richard H. Scheller at the above address.

Copyright (c) 1990 Society for Neuroscience $0270-6474 / 90 / 041380-08 \$ 02.00 / 0$
}

ular, this $13.0 \mathrm{kDa}$ protein is composed of an amino terminus rich in proline residues, a central hydrophilic core, and a hydrophobic carboxy terminus which presumably anchors the protein to the cytoplasmic face of the vesicles.

In a search for the mammalian homolog of this protein, we used low-stringency hybridization to screen rat brain cDNA libraries. Surprisingly, these screens revealed 2 classes of hybridizing clones. Sequence analysis confirmed that both are related to Torpedo VAMP and indicated that in the rat there are at least 2 VAMP genes (Elferink et al., 1989). The VAMP-1 and VAMP-2 genes encode proteins which differ only slightly in their primary sequence. As shown in Figure 1, the majority of amino acid differences between the 118 amino acid VAMP-1 and the 116 amino acid VAMP-2 are located in the proline-rich amino terminus and the hydrophobic carboxy terminus. Other significant amino acid changes may include the substitution in VAMP2 of leucine for the cysteine located just outside the membrane domain in VAMP-1. Preliminary RNA blotting analysis indicated that while both VAMP genes are expressed specifically in the rat nervous system, the 2 genes are expressed differentially within the brain and spinal cord (Elferink et al., 1989). The identification of 2 differentially expressed VAMP genes in the rat implies that the function of these proteins might be specialized for different neuronal types.

In this study, RNA blotting and in situ hybridization to rat brain was performed to precisely localize the differential sites of expression of the VAMP-1 and VAMP-2 genes. The results provide a detailed account of the distinct expression patterns of the 2 genes. VAMP-1 is primarily expressed in neurons involved in somatomotor function, while VAMP-2 expression is more generalized.

\section{Materials and Methods}

Antibodies and Western blots. The conserved hydrophilic core of rat VAMP-2 was subcloned as a Pst I to Bcl I restriction fragment into the bacterial expression vector pATH-11 (Dieckman and Tzagoloff, 1985) and expressed as a trpE fusion protein in $E$. coli. The fusion protein was purified by SDS-PAGE and excised from the gel. Homogenized gel slices were mixed with Freund's adjuvant (Sigma) and used to immunize New Zealand White rabbits. Serum was then affinity-purified by binding to, and eluting from, fusion protein bound to nitrocellulose. Serum purified in this way recognized only one band of the correct size in brain lysates from rat, Torpedo, and Discopyge ommata.

Western blots were performed essentially as described (Towbin et al., 1979). Protein, $10 \mu \mathrm{g}$, from each dissected brain region was separated by SDS-PAGE (Laemmli, 1970), transferred to nitrocellulose (Schleicher and Schuell, Keene, NH), and probed with affinity-purified antibody at $1 \mu \mathrm{g} / \mathrm{ml}$ final concentration. The blot was then probed with ${ }^{125} \mathrm{I}$-goat anti rabbit IgG (ICN Biomedicals, Lisle, IL), washed, and exposed at $-70^{\circ} \mathrm{C}$ with enhancement screens for $2 \mathrm{~d}$. 


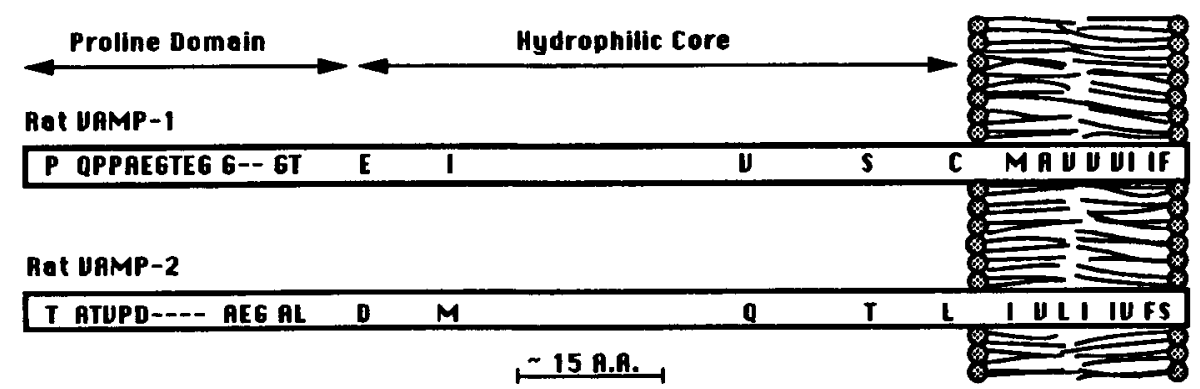

Figure 1. Schematic of rat VAMP-1 and VAMP-2. Amino acid differences between the 2 molecules, as deduced from cDNA sequences (Elferink et al., 1989), are shown and indicated by the single-letter amino acid code. Gaps introduced to maximize alignment between the 118 residue VAMP-1 and 116 residue VAMP-2 are indicated by a dash.
$R N A$ probes. RNA probes specific for either VAMP-1 or VAMP-2 were generated by subcloning cDNA fragments from their respective $3^{\prime}$ untranslated regions into Bluescript vector ks' (Stratagene, La Jolla, CA). The probe for rat VAMP-1 was an Xba I-Eco RI fragment corresponding to nucleotides 1179-1482 of cDNA clone RV1-1 (Elferink et al., 1989). The probe used for rat VAMP-2 was a Bam HI-Pst 1 fragment containing nucleotides 466-1090 for cDNA clone RV2-5. Antisense and sense RNA probes were transcribed using $T_{3}$ or $T_{7}$ RNA polymerase (Stratagene) to a specific activity of $1-2 \times 10^{7} \mathrm{cpm} / \mu \mathrm{g}$, according to the manufacturers specifications. All sequences are present in the GenBank/EMBL Data bank with the accession number J04827.

Northern blots. Total cellular RNA was prepared from dissected regions of female Sprague-Dawley rat brains by the $\mathrm{CsCl}$ /guanidine thiocyanate method of Chirgwin et al. (1979). Then, $15 \mu \mathrm{g}$ of total RNA from each section was denatured and electrophoresed through a $1 \%$ agarose gel containing $2.2 \mathrm{M}$ formaldehyde. The RNAs were transferred to Nytran (Schleicher and Schuell) nylon membranes and fixed by baking al $80^{\circ} \mathrm{C}$ for $1 \mathrm{hr}$. Filter hybridization was performed at $60^{\circ} \mathrm{C}$ according to the manufacturer's specifications using $3 \times 10^{5} \mathrm{cpm}$ of each antisense RNA probe per milliliter of hybridization solution, and final washes were performed in $30 \mathrm{~mm} \mathrm{NaCl}, 3$ mm sodium citrate, and $0.1 \%$ SDS at $65^{\circ} \mathrm{C}$ for $1 \mathrm{hr}$. The hybridization conditions used demonstrated no cross-reactivity between the probes specific for either VAMP-1 or VAMP. 2 . The integrity of the RNA prepared from the rat brain regions was verified by staining the filter with methylene blue (Zinn et al., 1983).

In situ histochemistry. Adult male Lewis rats (200-250 gm) were anesthetized with sodium pentobarbital $(40 \mathrm{mg} / \mathrm{kg})$ and were perfused transcardially with isotonic saline followed by $4.0 \%$ paraformaldehyde in 0.15 м phosphate buffer ( $\mathrm{pH} 7.8$ ). The brains were removed, postfixed for $4 \mathrm{hr}$ in the perfusion buffer, and cryoprotected by immersion in a series of cold, graded sucrose solutions to a final sucrose concentration of $25 \%$. The brains were frozen with dry ice and cut into $40-\mu \mathrm{m}$-thick coronal sections with a sliding microtome, collected in PBS, and mounted onto chrome alum/gelatin-subbed slides. Prehybridization treatments and hybridization conditions were essentially as described by Higgins et al. (1988). ${ }^{35}$ S-RNA transcripts were synthesized to a specific radioactivity of $6.6 \times 10^{8} \mathrm{cpm} / \mu \mathrm{g}$ from the $3^{\prime}$ untranslated regions of VAMP-1 and VAMP-2 in the Bluescript vector $\mathrm{ks}^{+}$(see above). To reduce background and enhance penetration of the probe, the RNA transcripts were reduced to fragments of approximately 100-200 nucleotides in length by hydrolysis with $0.2 \mathrm{~N} \mathrm{NaOH}$ at $0^{\circ} \mathrm{C}$ for $20-30$ min followed by neutralization with $2 \mathrm{M}$ HEPES and purification over Sephadex G50 NICK columns (Pharmacia). Prior to hybridization, the sections were treated with $4.0 \%$ paraformaldehyde in PBS for $5 \mathrm{~min}$ at room temperature, rinsed twice in PBS, and treated with proteinase $K$ (Boehringer-Mannheim) at $50 \mu \mathrm{g} / \mathrm{ml}$ in $5 \times \mathrm{TE}(1 \times \mathrm{TE}=10 \mathrm{mM}$ Tris $/ 1$ mM EDTA pH 8.0) for $7.5 \mathrm{~min}$. The tissue was rinsed with PBS and then dehydrated through a graded series of ethanol solutions containing $0.33 \mathrm{~mm}$ ammonium acetate. Slides were air-dried and then treated with a prehybridization mixture containing $50 \%$ formamide, $0.75 \mathrm{M}$ $\mathrm{NaCl}, 25 \mathrm{~mm}$ PIPES, pH 6.8, 25 mm EDTA, $5 \times$ Denhardt's solution, 0.25 M dithiothreitol, $0.2 \%$ SDS, $10 \%$ dextran sulfate (Pharmacia), and

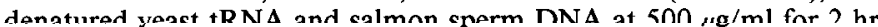
at $52^{\circ} \mathrm{C}$. For hybridization, $75-100 \mu \mathrm{l}$ of the same solution containing 7.5-10 $\mathrm{ng}$ of ${ }^{35} \mathrm{~S}$-labeled probe was applied to each slide, sealed under a coverslip, and incubated overnight at $52^{\circ} \mathrm{C}$. The coverslips were removed in $4 \times \mathrm{SSC}(1 \times \mathrm{SSC}=0.15 \mathrm{M} \mathrm{NaCl} / 15 \mathrm{~mm}$ sodium citrate, $\mathrm{pH}$ 7.0) containing $300 \mathrm{~mm} 2$-mercaptoethanol. Sections were treated with pancreatic RNase $(50 \mu \mathrm{g} / \mathrm{ml})$ in $0.5 \mathrm{M} \mathrm{NaCl} / 1 \times \mathrm{TE}$ for $30 \mathrm{~min}$ at $37^{\circ} \mathrm{C}$ and rinsed with $0.5 \times \mathrm{SSC}$ at $42^{\circ} \mathrm{C}$. The sections were air-dried, placed in film cartridges and exposed to DuPont Cronex x-ray film for $1-4 \mathrm{~d}$. Slides prepared for autoradiography were coated with NTB-2 emulsion (diluted 1:2 with $\mathrm{H}_{2} \mathrm{O}$ ), exposed 3-7 d, and developed with Kodak D. 19 developer. Alternate sets of tissue were stained with cresyl violet for identification of anatomical subregions.

\section{Results}

VAMP genes are differentially expressed

RNA was prepared from dissected regions of the rat brain and probed with single-stranded, ${ }^{32} \mathrm{P}$-labeled RNA probes synthesized using T7 or T3 polymerase (Fig. $2 A$ ). To reduce the likelihood of cross-hybridization between the homologous regions of the VAMP-1 and VAMP-2 cDNAs, RNA probes were generated to nonconserved $3^{\prime}$ untranslated sequences. When used individually, these probes detect bands of the same size as the corresponding coding sequence DNA probes. The different size of VAMP-1 and VAMP-2 messages permittcd mixing of the probes so that the relative quantities of each transcript could be easily visualized (Elferink et al., 1989). As can be seen in Figure $2 A$, the $2.4 \mathrm{~kb}$ VAMP-2 transcript appears abundant in all brain regions, with highest expression in the portions from the forebrain. In contrast, the $2.7 \mathrm{~kb}$ VAMP-1 transcript is only prevalent in the cerebellum, midbrain, brain stem, and spinal cord. Sense probes for both transcripts failed to hybridize to any transcripts.

Antibodies raised to the hydrophilic core of VAMP-2 expressed as a bacterial trpE fusion protein detected varying levels of VAMP proteins in all dissected rat brain regions tested (Fig. $2 B$ ). Highest expression was seen in the cortex, hippocampus, thalamus, and amygdala. Also, these antibodies cross-react with VAMP proteins in 2 divergent species of electric rays, namely, Torpedo californica and Discopyge ommata (not shown). Since T. californica VAMP is more homologous to rat VAMP-1 than rat VAMP-1 is to rat VAMP-2, it is therefore likely that these antibodies recognized both VAMP-1 and VAMP-2 proteins. As noted previously (Trimble et al., 1988), VAMP proteins run at anomolously low mobilities given their molecular size. On rare occasions the presence of a second, slightly lower mobility band in midbrain, brain stem, cerebellum, and spinal cord could be detected. Although this band could be VAMP-1, its resolution was not routinely reproducible.

\section{Regional expression of $V A M P-1$ and $V A M P-2$}

In order to more precisely localize the differential expression of VAMP-1 and VAMP-2 mRNAs, in situ hybridization of rat brain was performed using the RNA probes derived from $3^{\prime}$ untranslated regions (Figs. 4, 5). For both VAMP-1 and VAMP2 , the pattern of in situ hybridization was entirely consistent with the expression of either gene solely by neurons. For example, little or no signal above background was detected over 


\section{A}

K B

$$
12345678910111213
$$

9.5-

7.5-

4.4-

2.4-

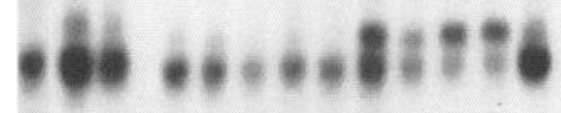

$1.4-$

B

KD

\section{$\begin{array}{llllllllllll}1 & 2 & 3 & 4 & 5 & 6 & 7 & 8 & 9 & 10 & 11 & 12\end{array}$}

$43.0-$

25.7-

18.4-

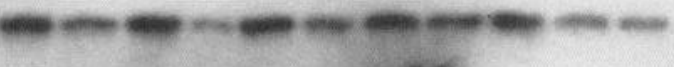

$14.3-$

6.2-

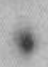

(1)

Figure 2. A, RNA blot analysis of dissected rat brain. Ten micrograms of total cellular RNA from olfactory bulb (lane 1), total brain (2), cortex (3), hippocampus (4), hypothalamus (5), thalamus (6), septum (7), amygdala (8), midbrain (9), cerebellum (10), brain stem (11), spinal cord (12), and residual portions (13) was blotted to nylon membrane and hybridized with an equal mixture of the 2 probes. $B$, Western blot analysis of dissected rat brain. Ten micrograms of protein from total brain (lane 1 ), olfactory bulb (2), cortex (3), pituitary (4), hippocampus (5), hiypothalamus (6), thalamus (7), septum (8), amygdala (9), midbrain (10), cerebellum (11), and spinal cord (12) was separated by SDSPAGE and transferred to nitrocellulose. The blot was probed with affinity-purified rabbit serum raised against rat VAMP-2 trpE-fusion protein and detected using ${ }^{125}$ I-labeled goat anti-rabbit secondary antibody.

major axon tracts such as the corpus callosum, the cerebral peduncles, the fornix, or the white matter of the cerebellum. Hybridization with the sense probe to VAMP-1 (Fig. 3) and VAMP-2 (not shown) showed only a low level of radioactivity reminiscent of Nissl staining, probably reflecting a background signal caused by high density of cell bodies.

Table 1 presents the regions of the brain and spinal cord where the highest levels of VAMP-1 and VAMP-2 mRNA hybridization were observed, and where the hybridization intensity was judged apparently higher for VAMP-1 or VAMP-2 or was equivocal (VAMP-1/VAMP-2). Table 1 was constructed by evaluating the data from both exposed X-ray film and emulsion-
Figure 3. Photomicrographs of X-ray film exposed by ${ }^{35} \mathrm{~S}$-labeled sense VAMP-1 probe hybridized to brain and spinal cord sections. Coronal sections, $40 \mu \mathrm{m}$, were hybridized to VAMP-1 sense RNA probe. The sections were arranged in a rostral to caudal arrangement left to right and top to bottom. The sections were then exposed on Dupont Cronex $\mathrm{X}$-ray film for $90 \mathrm{hr}$ before development. 


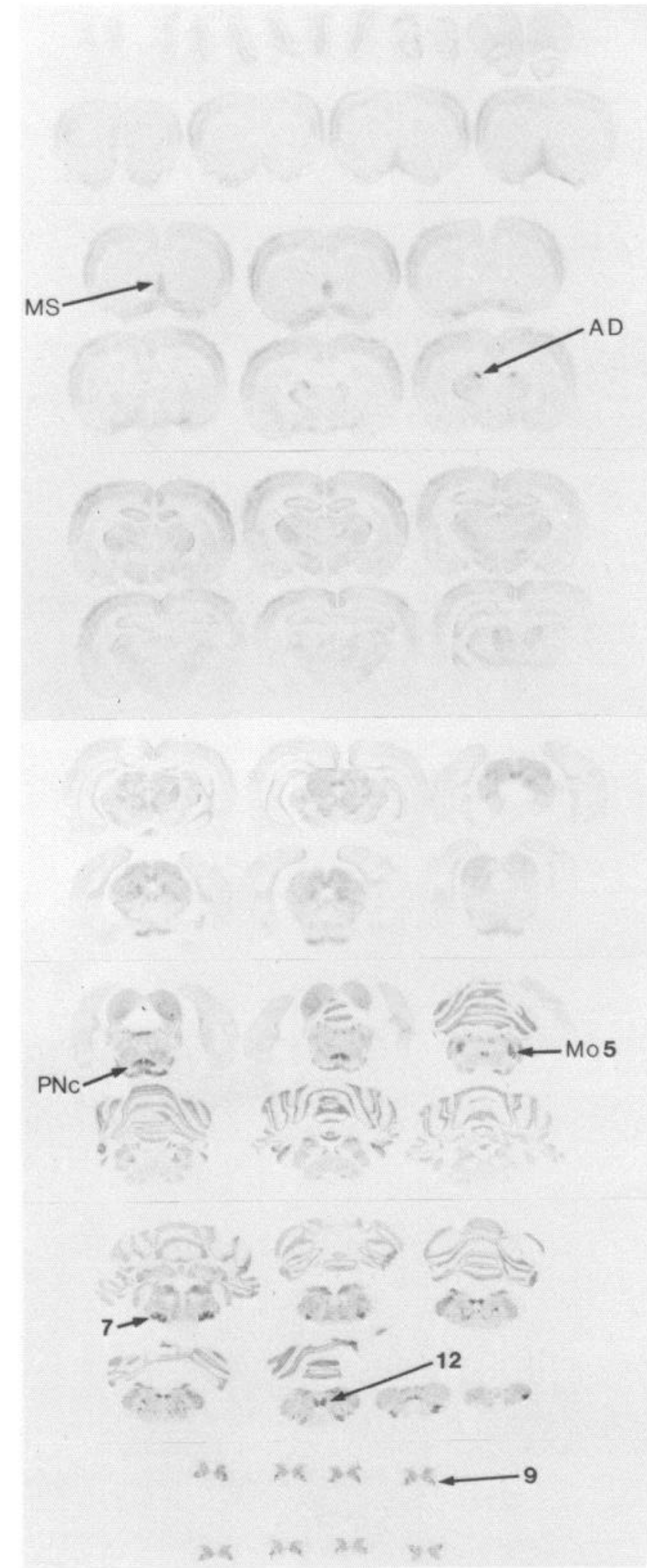

Figure 4. Photomicrographs of X-ray film exposed by ${ }^{35} \mathrm{~S}$-labeled antisense VAMP-1 RNA probes hybridized to brain and spinal cord sections as described in the legend to Figure 3. Abbreviations: $M S$, medial septum; $A D$, anterodorsal thalamic nucleus; $P N C$, pontine gray nuclei, pars caudalis; Mo5, motor nucleus of the trigeminal nerve; 7 , facial motor nucleus; 12 , hypoglossal motor nucleus; 9 , lamina 9 in the ventral horn of the spinal cord. Note that the last segment of spinal cord is in an inverted orientation. coated slides. The anatomical terminology is derived from the rat brain atlas of Paxinos and Watson (1982).

\section{Spinal cord}

Samples of the spinal cord were obtained from the cervical enlargement (approximately level C5) and just below the medullary-spinal junction. High levels of VAMP-1 mRNA were detected within the ventral horn as indicated by the exposure intensity on X-ray film (Fig. 4) and the high density of silver grains (Fig. 6A). VAMP-1 hybridization was especially apparent within the large motor neurons of lamina 9 (Figs. 4, 6A). Moderate to high levels of hybridization to VAMP-1 mRNA were observed within lamina 4-8 (Fig. 6A). The highest density of hybridization with the VAMP-2 probe was apparent within the dorsal horn, lamina 1-3 and 10 (Figs. 5, 6B). Less intense hybridization was also apparent within the ventral horn.

\section{Medulla}

VAMP-1 hybridization resulted in an intense signal within the lateral reticular formation and moderately intense within the cuneate, gracile, and external cuneate nucleus. Intense hybridization of the VAMP-1 probe was also apparent within the hypoglossal nucleus compared with the dorsal motor nucleus of the vagus nerve (Figs. 4, 6C). A moderately intense autoradiographic VAMP-1 signal indicating high levels of mRNA was apparent within the gigantocellular cells. In contrast, a high degree of VAMP-2 hybridization was present within the dorsal vagal motor nucleus (Figs. 5, 6D), the nucleus ambiguus, the inferior olive, and the superficial lamina of the spinal nucleus of $\mathrm{V}$, pars caudalis.

\section{Cerebellum and pons}

VAMP-1 and VAMP-2 hybridization level above background appeared within the cerebellar folia, although VAMP-1 hybridization intensity was significantly higher within neurons of the deep cerebellar nuclei. Both VAMP-1 and VAMP-2 probes exhibited moderate to high levels of hybridization within the locus coeruleus (Figs. 5, 6F), dorsal tegmental nucleus, and the pontine nuclei. Relatively dense hybridization of VAMP-1 was apparent within the VII, VI, and V cranial nerve motor nuclei, periolivary zones, trigeminal nuclei (Fig. 4). VAMP-2 probe hybridization was particularly apparent within parabrachial nuclei and the pontine central gray.

\section{Midbrain}

VAMP-2 hybridization appeared intense within the interpeduncular nucleus, central gray, parabigeminal nucleus (Fig. 5), dorsal Raphe' nuclei, medial terminal nucleus of the accessory optic tract, and substantia nigra, pars compacta (Fig. 5). VAMP1 hybridization was higher than VAMP-2 within the cranial nerve motor nuclei III and IV, the deep mesencephalic nucleus, the red nucleus, and the mesencephalic nucleus of V. Relatively equivalent hybridization levels were apparent within the superior and inferior colliculi.

\section{Diencephalon}

VAMP-1 hybridization was apparent within the anterior pretectal nucleus and the zona incerta. Overall, VAMP-2 hybridization levels were relatively high within most nuclei of the thalamus and hypothalamus. Particularly intense hybridization of VAMP-2 was observed within the medial preoptic region, supraoptic nucleus, ventromedial, paraventricular nucleus of 


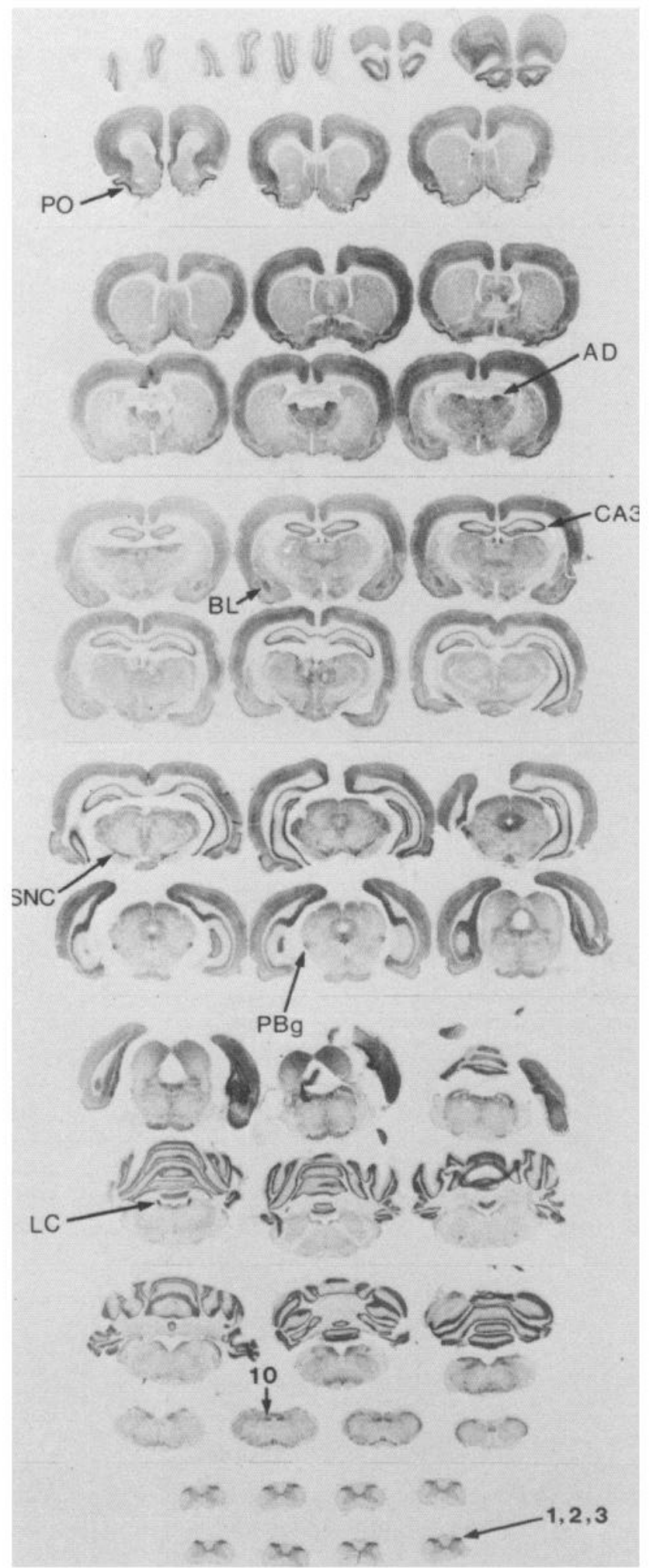

Figure 5. Photomicrographs of X-ray film exposed by ${ }^{35} \mathrm{~S}$-labeled antisense VAMP-2 RNA probe hybridized to brain and spinal cord sections as described in the legend to Figure 3. Abbreviations: $P O$, primary olfactory cortex; $A D$, anterodorsal thalamic nucleus; $B L$, basolateral amygdaloid nucleus; $C A 3$, CA3 region of the hippocampus; $S N C$, substantia nigra pars compacta; $P B g$, parabigeminal nucleus; $L C$, locus coeruleus; 10 , dorsal motor nucleus of the vagus nerve; $1,2,3$, lamina 1-3 in the dorsal horn of the spinal cord. (Paxinos and Watson, 1982). the hypothalamus, the parafascicular and medial geniculate nuclei of the thalamus, and medial habenula. Relatively equivalent hybridization for both probes was observed within the anterodorsal (Figs. 4, 5, 6E) and reticular thalamic nuclei.

\section{Telencephalon}

VAMP-1 hybridization was relatively high within the medial septal nucleus (Fig. 4), entopeduncular nucleus, and globus pallidus. VAMP-2 probe hybridization was generally higher within the telencephalon, especially within the neocortex, primary olfactory cortex (Fig. 5), presubiculum, CA3 region of hippocampus, nucleus of the lateral olfactory tract, and the basolateral amygdaloid nucleus (Fig. 5).

\section{Rhinencephalon}

Relatively low levels of VAMP-1 hybridization were present within the olfactory bulb. In contrast, VAMP-2 showed a high degree of hybridization within the olfactory bulb, especially within the internal plexiform, internal granular, and glomerular layers and the anterior olfactory nucleus.

\section{General comments}

In general, the in situ hybridization pattern of both VAMP probes was consistent with the data from Northern blots of RNA prepared from selected brain regions (Fig. $2 A$ ). the signals observed correspond well with what one would expect from summation of the in situ VAMP-1 and VAMP-2 signals in each of the dissected regions. For example, the VAMP-1 probe showed low levels of hybridization within the forebrain and higher levels of hybridization within the brain stem and spinal cord (Fig. 4). The VAMP-2 probe showed high levels of hybridization within most of the forebrain as compared to the brain stem and spinal cord (Fig. 5).

\section{Discussion}

The VAMP molecules represent an evolutionarily conserved group of synaptic vesicle proteins. The high degree of amino acid sequence conservation between the 2 rat forms and Torpedo VAMP has been described previously (Elferink et al., 1989). Recently, cloning of the cDNA encoding the bovine synaptic vesicle protein synaptobrevin has revealed that this molecule corresponds to a bovine homolog of VAMP (Südhof et al., 1989). In fact, only 2 conservative amino acid substitutions distinguish the bovine synaptobrevin/VAMP from rat VAMP-2: valine to alanine at amino acid position 8 and threonine to serine at amino acid position 116. In addition, the cloning of a Drosophila VAMP has revealed $78 \%$ amino acid conservation between the Drosophila and bovine proteins within the central core region of the molecule (Südhof et al., 1989). This high degree of homology quite likely underlines functional constraints on the structure of the central portion of the molecule.

The presence of 2 VAMP genes in rat, and probably in other mammals as well, suggests that the different forms might serve discrete functions within the brain. We demonstrate in this report that the VAMP-1 and VAMP-2 mRNAs are expressed in patterns which are distinct, but somewhat overlapping.

The use of $i n$ situ hybridization to localize the expression of synaptic vesicle proteins has significant advantage over the use of immunohistochemistry in that it identifies directly the cells which express the mRNA (and hence the protein) in contast to a more complex pattern of axonally transported protein localized or accumulating in nerve processes and distal nerve ter- 
Table 1. Localization of VAMP-1 and VAMP-2 mRNA expression in rat brain

VAMP-1

Lamina 4-9

Lateral reticular formation

Hypoglossal n.

Gracile, cuneate, and external cuneate $n$.

Deep cerebellar $\mathbf{n}$.

Facial n.

Periolivary zones

Abducens n.

Trigeminal motor $\mathrm{n}$.

Trigeminal sensory $n$.

Pontine n., caudal cochlear $\mathbf{n}$.

Red $\mathrm{n}$.

Oculomotor $\mathrm{n}$.

Trochlear $\mathrm{n}$.

Mesencephalic $\mathrm{n}$. of $\mathrm{V}$

Deep mesencephalic $n$.

Zona incerta

Entopeducular $\mathbf{n}$.

Mediai septal $n$.

Globus pallidus
VAMP-2

VAMP-1/VAMP-2

Spinal cord

Lamina $1-3,10$

Medulla

Inferior olivary $\mathbf{n}$.

Dorsal motor $\mathrm{n}$.

$\mathrm{N}$. ambiguus

Spinal trigeminal $n_{\text {., }}$ caudalis

Cerebellum and Pons

Parabrachial $\mathrm{n}$.

Pontine $\mathrm{n}$, pars caudalis

Pontine central gray

Pontine $\mathrm{n}$

Locus coeruleus

Dorsal tegmental $\mathbf{n}$.

Lateral dorsal tegmental $\mathbf{n}$.

Cerebellar folia

Midbrain
Parabigeminal $\mathrm{n}$.
Interpeduncular $\mathrm{n}$.
Dorsal Raphe $\mathrm{n}$.
Substantia nigra, pars compacta
Central gray
Medial terminal $\mathrm{n}$.
Diencephalon
Supraoptic n.
Ventromedial hypothalamus
Suprachiasmatic $\mathrm{n}$.
Medial preoptic region
Hypothalamic paraventricular $\mathrm{n}$.
Medial habenula
Medial geniculate $\mathrm{n}$.
Parafascicular n.
Telencephalon
Basolateral amygdala
Endopiriform $\mathrm{n}$.
CA3 hippocampus
N. lateral olfactory tract
Primary olfactory cortex
Presubiculum
Neocortex
Rhinencephalon
Olfactory bulb

Superior and inferior colliculi

Anterodorsal thalamic $\mathrm{n}$. Reticular thalamic $n$.



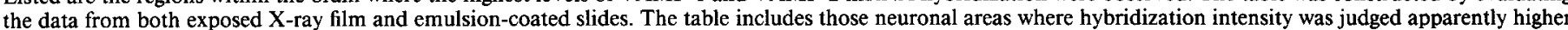

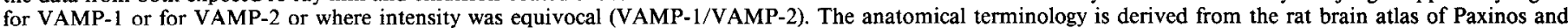
Watson (1982).
\end{abstract}

minals. Moreover, the ability to capitalize on the divergence of untranslated portions of the mRNA provides a means of dislinguishing the expression of the distinct VAMP-1 and VAMP2 genetic loci which encode very similar, but nonidentical, proteins. In fact, the previous report of immunohistochemistry of synaptobrevin/VAMP in the rat which involved the use of a monoclonal antibody (CL10.1) whose specific reactivity to either forms VAMP-1 or VAMP-2 was unknown. This VAMP antibody discriminated a pattern of expression that mirrored the distribution of ubiquitous synaptic vesicle proteins such as synaptophysin and synapsin I (Baumert et al., 1989). In addition to rat brain, CL10.1 stained synapses in the muscle layer of the stomach wall and the vas deferens, but not in synapses of skeletal muscle. Since this antibody selected VAMP-2 when used in immunopurification experiments, and since the expression of VAMP-1 suggests a role in neuromuscular transmission (see below), it is likely that the CL10.1 antibody recognizes an antigenic epitope present only on VAMP-2.

Overall, VAMP-1 is expressed predominantly in brain nuclei and spinal cord lamellae known to be involved in somatomotor function. High levels of expression were found in the motor nuclei of cranial nerves (III-VII, XII) which directly innervate muscles of the head, neck, face, jaw, and tongue. [Functional relationships discussed here are from Paxinos (1985).] High levels were also found in the cells of the ventral horn of the spinal cord (especially lamina 9) which innervate skeletal mus- 
Figure 6. Dark-field autoradiographic photomicrographs of emulsion-dipped sections hybridized to VAMP-1 $(A, C$, $E)$ and VAMP-2 $(B, D, F){ }^{35}$ S-labeled antisense RNA probes. $A$, Ventral horn cells of the spinal cord lamina 9 exhibiting hybridization to VAMP-1 antisense probe. $B$, Dorsal horn cells of the spinal cord lamina 1-3 exhibiting hybridization to VAMP-2 antisense RNA probe. $C$ and $D$, The region containing the hypoglossal, dorsal vagal, and solitary tract nuclei is shown. Neurons within the hypoglossal nucleus (12) exhibit hybridization to VAMP-1 antisense probe $(C)$, while neurons within the dorsal vagal nucleus $(10)$ hybridized with the VAMP-2 antisense probe $(D)$. $E$, Neurons within the anterodorsal thalamic nucleus hybridizing to VAMP-1 antisense probe. $F$, Neurons within the locus coeruleus hybridized with VAMP-2 antisense probe. Abbreviations: $v f u$, ventral funiculus; lf $u$, lateral funiculus; sol, nucleus of the solitary tract; $c c$, corpus callosum; $A D$, anterodorsal thalamic nucleus; $s m$, striatal medularis of the thalamus; $L C$, locus coeruleus; $I V$, fourth ventricle; $1,2,3$, spinal cord dorsal horn lamina $1-3 ; 9$, spinal cord ventral horn lamina $9 ; 10$, dorsal motor nucleus of the vagal nerve; 12 , hypoglossal nucleus. Scale bar, 250 $\mu \mathrm{m}$.
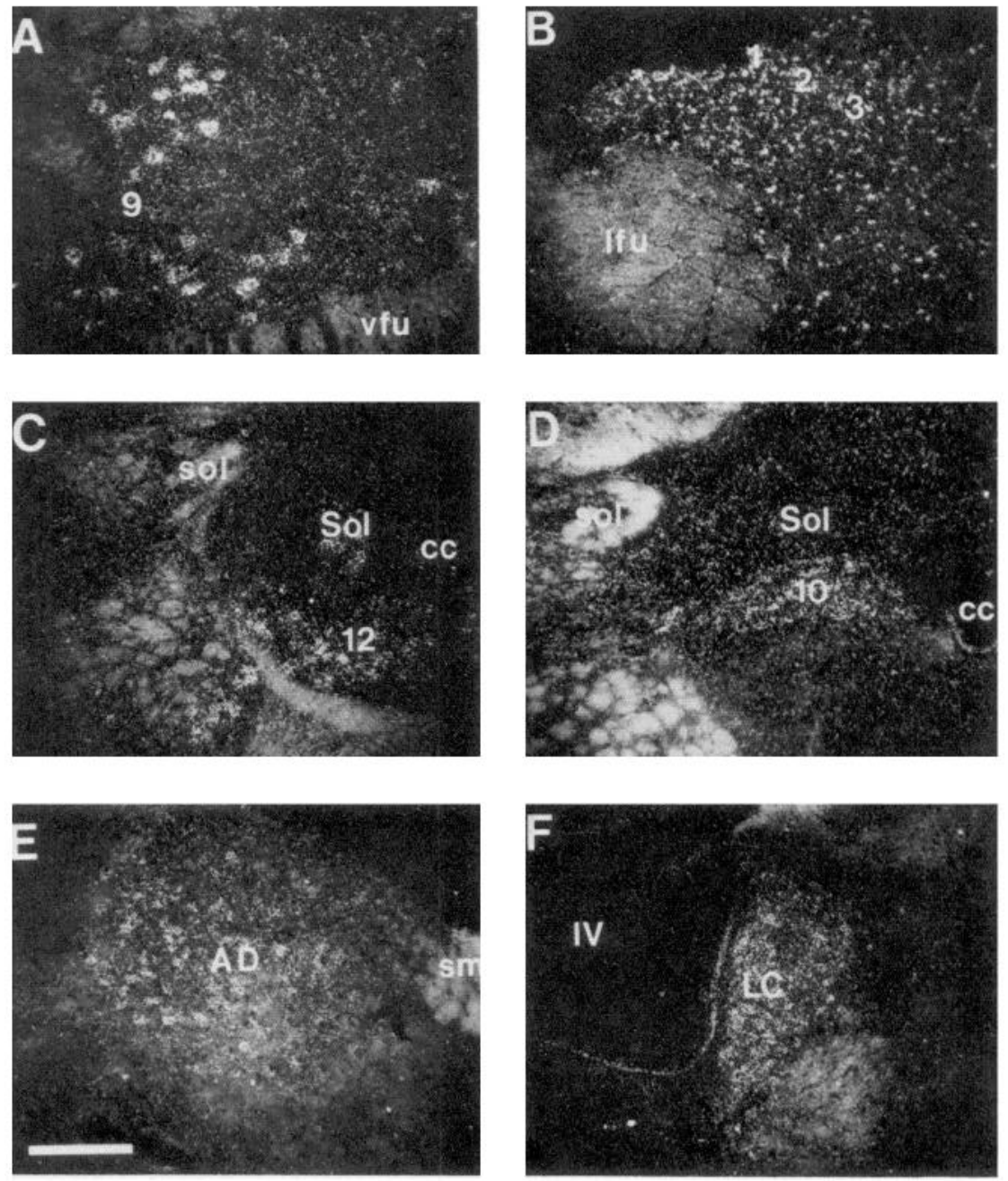

cles throughout the body. Many of the other nuclei hybridizing with VAMP-1, such as the red nucleus, modulate skeletal muscle activity indirectly by sending efferents to the ventral horn nuclei. In the case of the mesencephalic nucleus of $\mathrm{V}$, sensory inputs are relayed in a reflex arc via efferents to the motor nucleus of $\mathrm{V}$.

VAMP-2 is much more widely expressed, and predominates in nuclei responsible for autonomic motor, sensory, and integrative functions. Examples of the diverse expression of VAMP2 include the dorsal motor nucleus of the vagus nerve, which innervates the parasympathetic ganglia of the abdominal and thoracic viscera and ultimately contributes to the regulation of glands and visceral smooth muscle. High expression of VAMP2 is also found in the hypothalamic paraventricular and supraoptic nuclei, which are involved in autonomic and neuroendocrine functions (Swanson, 1987). VAMP-2 expression is also high in the substantia nigra pars compacta, which contains cells that have ascending dopaminergic projections into the frontal cortex, amygdala, and striatum.

VAMP-1 and VAMP-2 are both highly expressed in the locus coeruleus. This nucleus has roles in integrative and somatomotor processes. The majority of projections from this nucleus contain norepinephrine and innervate the hippocampus, neocortex, hypothalamus, and cerebellum. However, the major descending component innervates the ventral columns, interme- diate gray, and ventral half of the dorsal columns in the spinal cord.

One clear exception to the generalization that VAMP-1 and VAMP-2 expression patterns can be resolved along somatomotor and autonomic pathways, respectively, is the observation of VAMP-2 hybridization in the nucleus ambiguus, which contains large motoneurons innervating the laryngeal and pharyngeal muscles. However, this nucleus also contains parasympathetic motoneurons which innervate thoracic and abdominal viscera and other neurons which supply spinal respiratory motoneurons. It should be emphasized that in this and in many other nuclei, both forms were expressed, but the predominant form was as listed in Table 1 .

The expression of both forms of VAMP within single nuclei in the brain does not directly address the question of whether both proteins are expressed within the same cell. Although the contribution of each of the cell types within a nucleus to the final hybridization signal is not known, it does appear that in those areas in which both forms of VAMP are highly expressed, the same histological cell types seem to express both mRNAs. If single neurons do express both forms, it will be vital to determine if they provide similar or independent functions within those cells. Similarly, it will be of critical importance to establish if any neurons do not express either form of VAMP. Clearly, 
from the in situ experiments, it can be seen that many regions of the brain did not hybridize as strongly as others. Whether this can be accounted for on the basis of cell density or synaptic vesicle production, or whether it indicates a lack of VAMP production is unclear. At the present time we have evidence for only 2 forms of VAMP in the rat brain, but it is possible that other forms are synthesized in these loci. Alternatively, the presence of VAMP molecules may not be a requirement for the viability of all types of vesicles, and they may therefore be produced only in a subset of neurons.

Patterns of in situ hybridization similar to that observed for VAMP-1 have been previously described. The large neurofilament subunit (NF-II) in rat hybridizes intensely to motor nuclei in the medulla and pons but not at all in the diencephalon or telencephalon (Dautigny et al., 1988). Analogous hybridization patterns have been described for a new neuron-specific intermediate filament protein (peripherin) (Leonard et al., 1988). These proteins have both been proposed to play roles in stabilizing the neurofilaments of neurons possessing long axons. A comparable hybridization pattern has also been seen for the neuropeptide CGRP (Amara et al., 1985), which, like VAMP1 , is expressed in the hypoglossal and ventral motor nuclei but not the dorsal motor nuclei.

The precise function of the VAMP proteins within synaptic vesicles remains to be resolved. As yet, VAMP expression patterns do not appear to correlate directly with the neurotransmitter type, with the location of the nerve terminals within or outside of the CNS, or with the developmental history of the cells which cxpress them. However, the consistent expression of VAMP-1 in somatomotor neurons and in the neurons which innervate them suggests a possible functional relationship. The protein might be involved in some role specific to all of the cells within the motoneuron pathway. For example, if neuronal activity of these cells were significantly less than that of more active regions of the brain, then different cellular constraints would be placed on processes such as membrane recycling and perhaps even axonal transport. Furthermore, the cytoskeletal structures surrounding the active zones of such neurons could be significantly different. Through the further analysis of these molecules we hope to gain insights both into the molecular mechanisms of neurotransmission and possibly into the functional relationships within neuronal pathways in the nervous system.

\section{References}

Amara, S. G., J. L. Arriza, S. E. Leff, L. W. Swanson, R. M. Evans, and M. G. Rosenfeld (1985) Expression of a messenger RNA encoding a novel neuropeptide homologous to calcitonin gene-related peptide. Science 229: 1094-1097.

Baumert, M., P. R. Maycox, F. Navone, P. De Camilli, and R. Jahn (1989) Synaptobrevin: An integral membrane protein of 18,000 daltons present in small synaptic vesicles of rat brain. EMBO J. 8: 379384.

Buckley, K., and R. B. Kelly (1985) Identification of a transmembrane glycoprotein specific for secretory vesicles of neural and endocrine cells. J. Cell Biol. 100: 1284-1294.

Chirgwin, J. M., A. E. Przybyla, R. J. MacDonald, and W. J. Rutter
(1979) Isolation of biologically active ribonucleic acid from sources enriched in ribonuclease. Biochemistry 18: 5294-5299.

Dautigny, A., D. Pham-Dinh, C. Roussel, J. M. Felix, J. L. Nussbaum, and P. Jollès (1988) The large neurofilament subunit (NF-H) of the rat: cDNA cloning and in situ detection. Biochem. Biophys. Res. Commun. 154: 1099-1106.

Dieckmann, C. L., and A. Tzagoloff (1985) Assembly of the mitochondrial membrane system. CBP6, a yeast nuclear gene necessary for synthesis of cytochrome b. J. Biol Chem. 260: 1513-1520.

Elfcrink, L. A., W. S. Trimble, and R. H. Scheller (1989) Two vesicleassociated membrane protein genes are differentially expressed in the rat central nervous system. J. Biol Chem. 264: 11061-11064.

Floor, E., and B. E. Feist (1989) Most synaptic vesicles isolated from rat brain carry three membrane proteins, SV2, synaptophysin and p65. J. Neurochem. 52: 1433-1437.

Higgins, G. A., D. A. Lewis, S. Bahmanyar, D. Goldgaber, D. C. Gajdusek, W. G. Young, J. H. Morrison, and M. C. Wilson (1988) Differential regulation of amyloid-B protein $m R N A$ expression within hippocampal neuronal subpopulations in Alzheimer's disease. Proc. Natl. Acad. Sci. USA 85: 1297-1301.

Jahn, R., W. Schiebler, C. Ouimet, and P. Greengard (1985) A 38,000dalton membrane protein (p38) present in synaptic vesicles. Proc. Natl. Acad. Sci. USA 82: 4137-4141.

Kelly, R. B. (1988) The cell biology of the nerve terminal. Ncuron 1 : $431-438$.

Laemmli, U. K. (1970) Cleavage of structural proteins during the assembly of the head of bacteriophage T4. Nature 227: 680-685.

Leonard, D. G. B., J. D. Gorham, P. Cole, L. A. Greene, and E. B. Ziff (1988) A nerve growth factor-regulated messenger RNA encodes a new intermediate filament protein. J. Cell Biol. 106: 181-193.

Lowe, A. W., L. Madeddu, and R. B. Kelly (1988) Endocrine secretory granules and neuronal synaptic vesicles have three integral membrane proteins in common. J. Cell Biol. 106: 51-59.

Matthew, W. D., L. Tsavaler, and L. F. Reichardt (1981) Identification of a synaptic vesicle-specific membrane protein with a wide distribution in neuronal and neurosecretory tissue. J. Cell Biol. 91: 257269.

Obata, K., N. Kojima, H. Nishiyc, H. Inouc, T. Shirao, S. C. Fujita, and K. Uchizono (1987) Four synaptic vesicle-specific proteins: Identification by monoclonal antibodies and distribution in the nervous tissue and the adrenal medulla. Brain Res. 404: 169-179.

Paxinos, G. (1985) The Rat Nervous System, Vols. 1, 2, Academic, Sydney.

Paxinos, G., and C. Watson (1982) The Rat Brain in Stereotaxic Coordinates, Academic, Sydney.

Südhof, T., M. Baumert, M. S. Perin, and R. Jahn (1989) A synaptic vesicle membrane protein is conserved from mammals to Drosophila. Neuron 2: 1475-1481.

Swanson, L. W. (1987) The thalamus. In Handbook of Chemical Neuroanatomy. Integrated Systems of the CNS, Part I. Hypothalamus, Hippocampus, Amygdala and Retina, A. Bjorklund, T. Hökfelt, and L. W. Swanson, eds., pp. 1-124, Elsevier/North-Holland, Amsterdam.

Towbin, H., T. Staehelin, and J. Gordon (1979) Electrophoretic transfer of proteins from polyacrylamide gels to nitrocellulose sheets: Procedure and some applications. Proc. Natl. Acad. Sci. USA 76: 43504354.

Trimble, W. S., D. M. Cowan, and R. H. Scheller (1988) VAMP-1: A synaptic vesicle-associated integral membrane protein. Proc. Natl. Acad. Sci. USA 85: 4538-4542.

Wiedenmann, B., and W. W. Franke (1985) Identification and localization of synaptophysin, an integral membrane glycoprotein of $\mathrm{M}_{r}$ 38,000 characteristic of presynaptic vesicles. Cell 41: 1017-1028.

Zinn, K., D. DiMaio, and T. Maniatis (1983) Identification of two distinct regulatory regions adjacent to the human $\beta$-interferon gene. Cell 34: 865-879. 